(c) The Author(s) 2021. Published by Cambridge University Press on behalf of The Nutrition Society. This is an Open Access article, distributed under the terms of the Creative Commons Attribution licence (http://creativecommons.org/licenses/by/4.0/), which permits unrestricted re-use, distribution, and reproduction in any medium, provided the original work is properly cited.

\title{
Metabolic and molecular signatures of improved growth in Atlantic salmon (Salmo salar) fed surplus levels of methionine, folic acid, vitamin $B_{6}$ and $B_{12}$ throughout smoltification
}

\author{
Anne-Catrin Adam ${ }^{1 *}$, Takaya Saito ${ }^{1}$, Marit Espe ${ }^{1}$, Paul Whatmore ${ }^{1} \uparrow$, Jorge Manuel De Oliveira Fernandes ${ }^{2}$, \\ Vibeke Vikeså ${ }^{3}$ and Kaja H. Skjærven ${ }^{1}$ \\ ${ }^{1}$ Institute of Marine Research, 1870 Nordnes, 5817 Bergen, Norway \\ ${ }^{2}$ Nord University, Faculty of Biosciences and Aquaculture, 1490, 8049 Bodø, Norway \\ ${ }^{3}$ Skretting ARC, Stavanger, 48, 4001 Stavanger, Norway
}

(Submitted 23 February 2021 - Final revision received 14 May 2021 - Accepted 18 June 2021 - First published online 25 June 2021)

\section{Abstract}

A moderate surplus of the one carbon (1C) nutrients methionine, folic acid, vitamin $\mathrm{B}_{6}$ and $\mathrm{B}_{12}$ above dietary recommendations for Atlantic salmon has shown to improve growth and reduce hepatosomatic index in the on-growing saltwater period when fed throughout smoltification. Metabolic properties and molecular mechanisms determining the improved growth are unexplored. Here, we investigate metabolic and transcriptional signatures in skeletal muscle taken before and after smoltification to acquire deeper insight into pathways and possible nutrient-gene interactions. A control feed (Ctrl) or 1C nutrient surplus feed $(1 \mathrm{C}+$ ) were fed to Atlantic salmon 6 weeks prior to smoltification until 3 months after saltwater transfer. Both metabolic and gene expression signatures revealed significant $1 \mathrm{C}$ nutrient-dependent changes already at pre-smolt, but differences intensified when analysing post-smolt muscle. Transcriptional differences revealed lower expression of genes related to translation, growth and amino acid metabolisation in post-smolt muscle when fed additional $1 \mathrm{C}$ nutrients. The 1C+ group showed less free amino acid and putrescine levels, and higher methionine and glutathione amounts in muscle. For Ctrl muscle, the overall metabolic profile suggests a lower amino acid utilisation for protein synthesis, and increased methionine metabolisation in polyamine and redox homoeostasis, whereas transcription changes are indicative of compensatory growth regulation at local tissue level. These findings point to fine-tuned nutrient-gene interactions fundamental for improved growth capacity through better amino acid utilisation for protein accretion when salmon was fed additional 1C nutrients throughout smoltification. It also highlights potential nutritional programming strategies on improved post-smolt growth through 1C+ supplementation before and throughout smoltification.

Key words: Muscle: Micronutrients: One-carbon metabolism: RNA-sequencing: Metabolomics

In Atlantic salmon (Salmo salar) farming, the feed currently consists of $60 \%$ plant-based material, which is a substantial increase from $22 \%$ in $2000^{(1)}$. The change in feed raw materials from marine to plant based alters the requirement levels of certain micronutrients. Recommendations have thereby been re-evaluated through the EU-funded ARRAINA project ${ }^{(2-6)}$, and recent research also points to methionine as a key micronutrient along with folic acid, vitamin $\mathrm{B}_{6}$ (pyridoxine) and vitamin $\mathrm{B}_{12}$ (cobalamin) for improved and healthy growth through smoltification $^{(7,8)}$. B vitamin supplementation above the NRC recommendations improved overall growth performance, protein retention and reduced relative liver size of Atlantic salmon ${ }^{(3,4,6,9,10)}$. Dietary micronutrients including the B vitamins have been proposed as the most probable candidates affecting fillet texture and increasing muscle cell size observed in post-smolts ${ }^{(5)}$.

Methionine, folic acid, vitamin $\mathrm{B}_{6}$ and vitamin $\mathrm{B}_{12}$ are important substrates and cofactors in the one-carbon (1C) metabolism, hereinafter collectively referred to as $1 \mathrm{C}$ nutrients. The $1 \mathrm{C}$ metabolism links several pathways that allocate 1C groups by S-adenosylmethionine (SAM) that is synthesised from methionine by the enzyme methionine adenosyltransferase. In mammals, SAM affects not only energy metabolism, synthesis of proteins, phosphatidylcholines, creatine and polyamines but

Abbreviations: Ctrl, control feed; GH, growth hormone; GHR, growth hormone receptor; GSH, glutathione; IGF, insulin-like growth factor; IGFBP, insulin-like growth factor binding protein; ORA, over-representation analysis; SAH, S-adenosylhomocysteine; SAM, S-adenosylmethionine; 1C, one carbon.

* Corresponding author: Anne-Catrin Adam, email Anne-Catrin.Adam@hi.no

$\dagger$ Present address: eResearch Office, Queensland University of Technology, 2434, Brisbane, QLD 4001, Australia 
also redox defence, cell signalling through post-translational protein modification and epigenetic control of gene expression through methylation of DNA and histones ${ }^{(11-15)}$. S-adenosylhomocysteine (SAH) is formed after donation of the methyl group from SAM to a methyl acceptor and hydrolysed to homocysteine. Homocysteine can be re-methylated back to methionine by methionine synthase that requires vitamin $\mathrm{B}_{12}$ and 5-methyltetrahydrofolate, re-methylated through the betaine homocysteine methyltransferase pathway or irreversibly transsulphurated through the vitamin $\mathrm{B}_{6}$-dependent cystathionine $\beta$-synthase to glutathione $(\mathrm{GSH}){ }^{(16,17)}$

Skeletal muscle mass represents a dynamic balance between protein synthesis and degradation controlled by numerous factors, including growth hormone $(\mathrm{GH})$, insulin-like growth factors (IGF-1, IGF-2) and their cell surface receptors, and IGF-binding proteins (IGFBP) in the GH/IGF system in fish ${ }^{(18-21)}$. A shift towards increased protein synthesis leads to positive protein accretion, and the availability of nutrients is one of the most important factors influencing muscle growth performance in fish ${ }^{(22)}$. GH activates IGF-1 production through GH receptors (GHR) mainly in liver, whereas IGF function greatly depends on IGFBP activity in target tissues such as muscle ${ }^{(19,23)}$. During protein synthesis in muscle, myofibrillar content increases along with the enlargement of muscle fibres. The formation of muscle tissue, called myogenesis, is regulated by several myogenic regulatory factors and myocyte-specific enhancer factor 2 transcription factors that stimulate differentiating myocytes to fuse to multinucleated myofibres during myofibrillogenesis ${ }^{(20,24,25)}$. Endocrine regulation of growth, particularly circulating plasma GH levels are nutritionally regulated, however, nutritional regulation of IGFBP receptors and GHR is tissue specific that remains to be understood ${ }^{(26)}$. The availability of certain nutrients such as lysine, methionine or B vitamins through the diet has been shown to modulate GH/IGF responses by modulating gene expression and protein deposition, turnover thus affecting growth performance in fish ${ }^{(7,27,28)}$. The crosstalk between the GH/IGF system and sirtuins, which de-acylate histones or non-histone proteins, is coupled to the energy status of a cell via $\mathrm{NAD}+$ and is involved in tuning growth energy-demanding processes $^{(26)}$.

Factors such as photoperiod ${ }^{(29)}$, temperature ${ }^{(30-32)}$, circadian clock $^{(33)}$, dietary macronutrients such as protein, but also dietary micronutrients ${ }^{(3,5,7,10,16,34)}$ affect muscle growth and quality in teleost species. The role of dietary $1 \mathrm{C}$ nutrients when feeding plant-based feed has broad consequences for amino acid availability utilised for growth ${ }^{(16)}$, for regulating mRNA levels of genes encoding enzymes in the $1 \mathrm{C}$ metabolism both intra- ${ }^{(10)}$ and intergenerationally ${ }^{(17)}$, and moreover it has shown to enhance growth by altering the metabolism in both liver and muscle of Atlantic salmon $^{(7,35)}$. In a recently published study, two $1 \mathrm{C}$ nutrient surplus levels were given in a Med-1C and a High-1C diet on top of the current recommendations as formulated in the Low-1C $\operatorname{diet}^{(7)}$. When salmon was fed the highest surplus level (High1C), no additional benefit for growth was reported in the respective trial. Feeding a moderate surplus of $1 \mathrm{C}$ nutrients (Med-1C) during the fresh water period, through smoltification until the on-growing saltwater period, increased body weight and decreased liver weight of salmon, which was reflected in a higher condition factor and specific growth rate compared with the Low-1C group $^{(7)}$. The present study compares muscle from Low-1C and Med-1C fed salmon, hereinafter named as Ctrl and $1 \mathrm{C}+$, respectively. Describing the metabolic signatures and gene expression profiles in muscle from better growing $1 \mathrm{C}+$ fed salmon will improve our knowledge on active pathways and interactions to explain how surplus $1 \mathrm{C}$ nutrients fed to pre-smolts throughout smoltification improves growth already at the start of the on-growing saltwater period.

\section{Materials and methods}

\section{Feeding trial}

The Atlantic salmon feeding trial was performed at Lerang Research Station (Skretting Aquaculture Research Centre). In accordance to Norwegian Regulation on Animal Experimentation (FOR-2015-06-18-761) and European legislation (Directive 2010/ $63 / \mathrm{EU}$ ), formal ethical approval of the experiment by the Norwegian Animal Research Authority was not required as experimental conditions were practices undertaken for the purpose of recognised animal husbandry, and experimental feed was not deficient in nutrient composition with regard to health and welfare of the animals. Two earlier described experimental feeds ${ }^{(7)}$, Ctrl (Low-1C) and 1C+ (Med-1C), contained varying levels of methionine, folate, vitamin $\mathrm{B}_{6}$ and $\mathrm{B}_{12}$, collectively named as $1 \mathrm{C}$ nutrients. Analysed proximate composition and $1 \mathrm{C}$ nutrient levels in the feed are given in Table 1. Feed composition is provided in online Supplementary Table S1 and Fig. S1. For the Ctrl feed, $\mathrm{B}$ vitamins were included at recommended levels ${ }^{(6)}$ and methionine at known NRC requirement levels ${ }^{(9)}$. The $1 \mathrm{C}+$ feed contained a moderate surplus of four $1 \mathrm{C}$ nutrients to support maximal performance as suggested in previous work ${ }^{(6,36)}$.

Primary outcomes of the feeding experiment, that is, growth performance, body indices, nutrient retention, feed conversion ratio and protein utilisation of the same experimental fish, were described by Espe et $a l .{ }^{(7)}$.

The feeds were fed in triplicate tanks 6 weeks prior to smoltification until 3 months after saltwater transfer (Fig. 1). Pre-smolt salmon (SalmoBreed strain) was randomly assigned to experimental tanks for a 2 -week long acclimatisation period prior to

Table 1. Analysed proximate composition and $1 \mathrm{C}$ nutrient levels in $\mathrm{Ctrl}$ and $1 \mathrm{C}+$ feed. Re-used and modified table from Espe et al. ${ }^{(7)}$. Feed composition is provided in online Supplementary Table S1

\begin{tabular}{|c|c|c|c|c|c|}
\hline & \multirow[b]{2}{*}{ Base* } & \multicolumn{2}{|c|}{$3 \mathrm{~mm}$} & \multicolumn{2}{|c|}{$4 \mathrm{~mm}$} \\
\hline & & Ctrl & $1 \mathrm{C}+$ & Ctrl & $1 \mathrm{C}+$ \\
\hline \multicolumn{6}{|c|}{ Analysed composition } \\
\hline Crude protein $(\mathrm{g} / \mathrm{kg})$ & $436 \cdot 7 \dagger$ & - & - & - & - \\
\hline Crude lipid (g/kg) & $235 \dagger$ & - & - & - & - \\
\hline $\mathrm{DM}(\mathrm{g} / \mathrm{kg})$ & 920 & - & - & - & - \\
\hline Energy (MJ/kg) & $22.8 †$ & - & - & - & - \\
\hline Vitamin $\mathrm{B}_{12}(\mathrm{mg} / \mathrm{kg})$ & & 0.16 & $0 \cdot 18$ & 0.15 & 0.16 \\
\hline Folate $(\mathrm{mg} / \mathrm{kg})$ & & $2 \cdot 90$ & 4.80 & $2 \cdot 60$ & 4.60 \\
\hline Vitamin $B_{6}(\mathrm{mg} / \mathrm{kg})$ & & $6 \cdot 75$ & 8.45 & $7 \cdot 01$ & $9 \cdot 31$ \\
\hline Methionine $(\mathrm{g} / \mathrm{kg})$ & & $6 \cdot 7$ & $9 \cdot 2$ & $6 \cdot 7$ & 9.5 \\
\hline
\end{tabular}

* Base values indicate that all values in the same row are equivalent/similar across the feeds and the pellet sizes.

$\dagger$ Values are not equivalent but similar as determined by their relatively small SD: $\pm 5 \cdot 2$, \pm 5.5 and \pm 0.18 , respectively. 


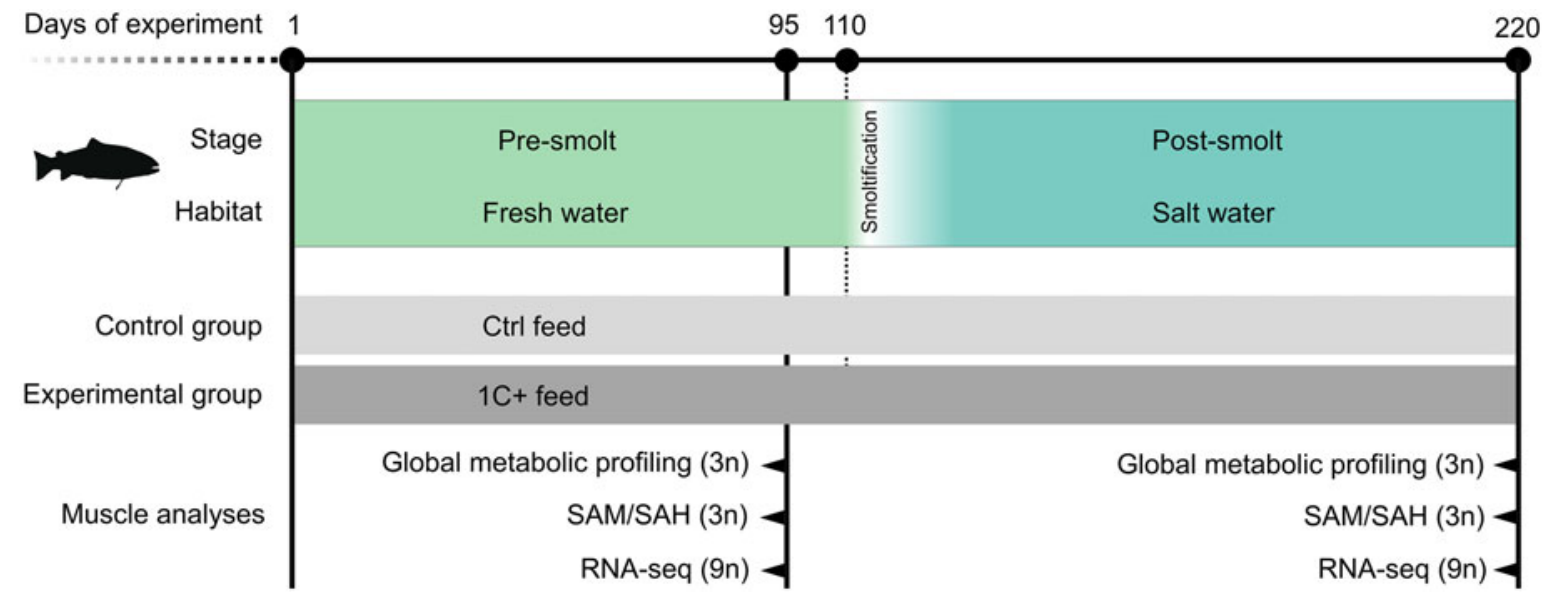

Fig. 1. Experimental design and sampling of muscle from Atlantic salmon fed a control feed (Ctrl) and a feed including surplus levels of one-carbon (1C) nutrients (1C + ) through the smoltification period. The $\mathrm{Ctrl}$ feed contained $1 \mathrm{C}$ nutrients (folate, methionine, vitamin $\mathrm{B}_{6}$ and $\mathrm{B}_{12}$ ) as recommended and on the requirement levels to support maximal performance (ARRAINA(6) and $\mathrm{NRC}^{(9)}$ ). The $1 \mathrm{C}+$ feed included even higher levels of the $1 \mathrm{C}$ nutrients, which improved growth when given through smoltification $^{(7)}$. Fish were fed in triplicate tanks for 6 weeks in the fresh water period, through saltwater transfer and 3 months in the on-growing saltwater period. Muscle tissue was sampled in the end of the fresh water and in the on-growing saltwater period for further analyses: global metabolic profiling, SAM/SAH by HPLC and RNA-sequencing (RNA-seq). Figure is modified from Espe et al. ${ }^{(7)}$.

the start of the experiment as earlier described ${ }^{(7)}$. Tanks from all treatment groups were placed in random order. To avoid poor tank environment, abnormal behaviour and poor welfare, ninety fish per tank were initially kept during the freshwater period (mean body weight of $32 \cdot 1 \mathrm{~g}$ (bulk weight), tank diameter $0.6 \mathrm{~m}$, tank volume $70 \mathrm{l}$, water flow 300-450 l/h; rearing temperature $\left.11.9(\mathrm{sD} 0.4)^{\circ} \mathrm{C}\right)$, and then moved to larger tanks for the saltwater period (mean body weight of 95.0 (sD 3.9) g, tank diameter $0.6 \mathrm{~m}$, tank volume $450 \mathrm{l}$, water flow $450-900 \mathrm{l} / \mathrm{h}$; rearing temperature $\left.12 \cdot 0(\mathrm{SD} 0 \cdot 1)^{\circ} \mathrm{C}\right)^{(7)}$. Fish were fed continuously until day 94 of the experiment, and thereafter fed three meals daily for $2 \mathrm{~h}$ per meal $(8 \mathrm{am}, 12 \mathrm{am} \text { and } 8 \mathrm{pm})^{(7)}$. The fish received $12 \mathrm{~h}$ light and $12 \mathrm{~h}$ darkness during the first 6 weeks, and continuous light for the rest of the experiment ${ }^{(7)}$. Fish were vaccinated at day 38 and starved for $5 \mathrm{~d}$ during vaccination. Early signs of disease, pain or distress were regularly monitored to avoid suffering. $40 \mathrm{mg} / \mathrm{l}$ tricaine (Pharmaq) was used to anaesthetise fish before all handling, and samples were collected from salmon $24 \mathrm{~h}$ after feeding to address effects on metabolism rather than reflecting the feed itself $^{(7)}$. Tricaine solved in salt water requires no additional buffering, but tricaine solved in fresh water was buffered with $40 \mathrm{mg} / \mathrm{l} \mathrm{Na} \mathrm{CO}_{3}$.

\section{Pre-smolt and post-smolt muscle sampling}

Fast muscle from Ctrl and $1 \mathrm{C}+$ fed salmon were taken in the end of the fresh water period (pre-smolt, day 95) and in the on-growing saltwater period (post-smolt, day 220) as illustrated in Fig. 1. Muscle tissue was obtained from the same epaxial area in the filet between dorsal and anal fin from three fish of each of the three replicate tanks per group. mRNA was extracted from muscle from individual fish for RNA-sequencing (online Supplementary Table S2). Global metabolic profiling and $\mathrm{SAM} / \mathrm{SAH}$ analysis were performed on the same pooled samples containing muscle tissue from five fish derived from the same tank (online Supplementary Table S3).

\section{S-adenosylmethionine and S-adenosylhomocysteine analysis}

SAM and SAH were determined in twelve pooled muscle samples (each contained five muscles from fish of the same tank) on a reverse phase HPLC after deproteinisation in $0.4 \mathrm{M} \mathrm{HClO}_{4}$ and quantified using standards of the respective metabolites (Sigma) as earlier described ${ }^{(35,37)}$

\section{Global metabolic profiling}

Twelve muscle samples containing pooled muscle tissue of five individual fish from the same tank were analysed in an $\mathrm{MxP}^{\circledR}$ Global Profiling by Metanomics Health GmbH. Samples were weighed, freeze dried and homogenised, and dry weight was determined before extraction. After extraction and protein precipitation, samples were separated into lipid and polar fractions before further analysis using the GC-MS (Agilent 6890 GC coupled to an Agilent 5973 MS System, Agilent) and liquid chromatography-MS/MS (LC-MS/MS; Agilent 1100 HPLCSystem, Agilent, coupled to an Applied Biosystems API4000 MS/MS-System, Applied Biosystems). Integration and validation of chromatographic data were performed by Metanomics Health $\mathrm{GmbH}$. Metabolite levels were normalised against the median of the pool reference samples (derived from aliquots of all samples) to give pool-normalised ratios performed for each sample per metabolite. GraphPad Prism (version 8.3.0) was used for illustration of pool-normalised metabolite data. Evaluation of all metabolites was semi-quantitative and metabolite levels were reported as pool-normalised ratios (online Supplementary Table S4). Results were given for free (non-covalently bound) metabolites as sample preparation did not involve hydrolysation.

\section{mRNA extraction}

For mRNA extraction, muscle tissue of thirty-six individual fish was homogenised each in Qiazol (Qiagen) and three ceramic beads (CK28) using a Precellys 24 homogeniser (Bertin 
Technologies) at $6000 \mathrm{rpm}$. Total RNA was extracted following the EZ1 RNA Tissue Mini Kit (Qiagen) including a DNAase treatment using the RNAse-Free DNAse set (Qiagen). RNA quantity and quality were verified using NanoDrop ${ }^{\circledR}$ ND-1000 Spectrophotometer (NanoDrop Technologies) and Agilent 2100 Bioanalyser (RNA 6000 Nano LabChip kit, Agilent Technologies), respectively.

\section{RNA library preparation and high-throughput RNA-sequencing}

Total RNA quality was assessed before creating mRNA fragments for high-throughput sequencing (RNA-seq). RNA integrity number (RIN) values from a total of thirty-six samples were on average $9 \cdot 3$ (RINmin $=8 \cdot 2$; RINmax $=9 \cdot 7$, online Supplementary Table S2). The libraries were divided into two sets of eighteen samples each, which were prepared simultaneously and were balanced for sex, dietary group and stage. See online Supplementary File S1 for library preparation and sequencing details. The generated libraries of an approximately $200 \mathrm{nt}$ insert size were PCR enriched, and quality of the libraries was assessed; they were barcoded, pooled and denatured according to the NextSeq System Denature and Dilute Libraries Guide (Illumina). The two library pools were sequenced on a NextSeq500 (Illumina) at Nord University to generate 76 bp single-end reads. Fastqc v0.11.8 (Babraham Bioinformatics) was used for quality assessment. Quality control assessment indicated that sequences were of high quality with minimal adapters present and trimming was not required. Quality-trimmed reads were mapped to the latest Atlantic salmon RefSeq reference genome and annotation (ICSASG_v2. 6/10/2016). This genome was indexed using the latest version of HISAT2 (v2.1.0).

\section{Statistical analyses}

Differences in metabolites between treatment groups (pre-smolt $1 \mathrm{C}+v$. pre-smolt $\mathrm{Ctrl}$, and post-smolt $1 \mathrm{C}+v$. post-smolt Ctrl) were determined by calculating the ratio of the group means from log10-transformed data for each of the metabolites (online Supplementary Table S4). Significantly different metabolites in each group comparison were determined using ANOVA with Tukey adjustment and false discovery rate by Benjamini and Hochberg multiple comparison adjustment ${ }^{(38)}$. The enrichment for significant altered metabolites (false discovery rate $<0 \cdot 1$ ) in a metabolite class shows the largest differences in the metabolic profiles using enrichment scores (online Supplementary Table S5). One score corresponds to the ratio between the odds of the number of altered metabolites among the number of detected metabolites in one class and the odds of all altered metabolites among all detected in the whole screening. The significance of enrichment (adjusted $P<0.05$ ) was assessed by Fisher's exact test with Benjamini and Hochberg multiple comparison correction ${ }^{(38)}$ using the functions fisher.test and p.adjust in R, respectively.

Statistically significant changes in SAM and SAH levels comparing the $1 \mathrm{C}+$ with the $\mathrm{Ctrl}$ group were assessed by an unpaired $t$ test (two-tailed) with $P<0 \cdot 05$. F test and Shapiro-Wilk test were applied to test variance and normality of the data.

For RNA-seq analysis, differential gene expression analysis was determined using the $\mathrm{R}$ package DESeq2 with default parameters (version 1.24.0) that estimates variance-mean dependence in count data from high-throughput sequencing assays and tests for differential expression based on a model using the negative binomial distribution ${ }^{(39)}$. Two differential expression comparisons were made: (1) pre-smolt $1 \mathrm{C}+v$. pre-smolt Ctrl and (2) post-smolt $1 \mathrm{C}+v$. post-smolt Ctrl. Statistical significance of over-representation of differentially expressed genes (DEG, $|\log 2 \mathrm{FC}|>1$ and adjusted $P<0.05$ ) in KEGG pathways and gene ontology terms was determined using a hypergeometric distribution test (over-representation analysis (ORA)) using the package clusterProfiler ${ }^{(40)}$. Since ORA is ratio based, many genes can be annotated to multiple pathways and these tests can also lead to false positives when species and pathways are poorly annotated. Gene Set Enrichment Analysis relies on the whole set of genes detected, allows the detection of smaller differences and being able to indicate either up- or down-regulation for pathways instead of selecting DEG based on fold change thresholds for defining differences as in case of ORA ${ }^{(10,41)}$. Both ORA and Gene Set Enrichment Analysis were performed to examine enrichment in KEGG pathways.

No data points or samples were excluded in the analysis of metabolites, SAM and SAH, or RNA-seq.

\section{Analysis and bioinformatics environment}

For metabolomics and SAM/SAH analyses, the statistical software R (version 3.4.4) and GraphPad Prism 8.3.0 (GraphPad Software) were used. RNA-seq analysis was completed using Linux tools (online Supplementary File S1) and bioconductor packages for downstream analysis using the $\mathrm{R}$ programming language version 3.6.1 (2019-07-05, https://www.r-project.org/).

\section{Data availability}

Unaligned RNA-seq raw data are accessible through accession number PRJNA680206 (https://www.ncbi.nlm.nih.gov/sra/). Supplementary tables have been made available on figshare (https://doi.org/10.6084/m9.figshare.14484489.v1).

\section{Results}

\section{Metabolic and transcriptomic changes in pre-smolt and post-smolt muscles}

Metabolic profiles from both Ctrl and $1 \mathrm{C}+$ muscle were analysed using a mass spectrometric-based metabolomic approach, where in total 536 free, non-covalently bound metabolites were detected (online Supplementary Table S4). The most significantly different metabolites were found in post-smolt (thirtyseven metabolites) than in pre-smolt (thirteen metabolites) muscle when comparing $1 \mathrm{C}+$ and Ctrl groups (Fig. 2(a)). However, for both pre- and post-smolts, the differences between the dietary groups were found in the free amino acid profile and their conjugates, and again the results show that this effect was less pronounced in pre-smolt than in post-smolt muscle (Fig. 2(b) and online Supplementary Table S5).

For RNA-seq analysis, on average $94.2 \%$ of 24.6 million reads were mapped to the reference genome (online Supplementary 
(a)

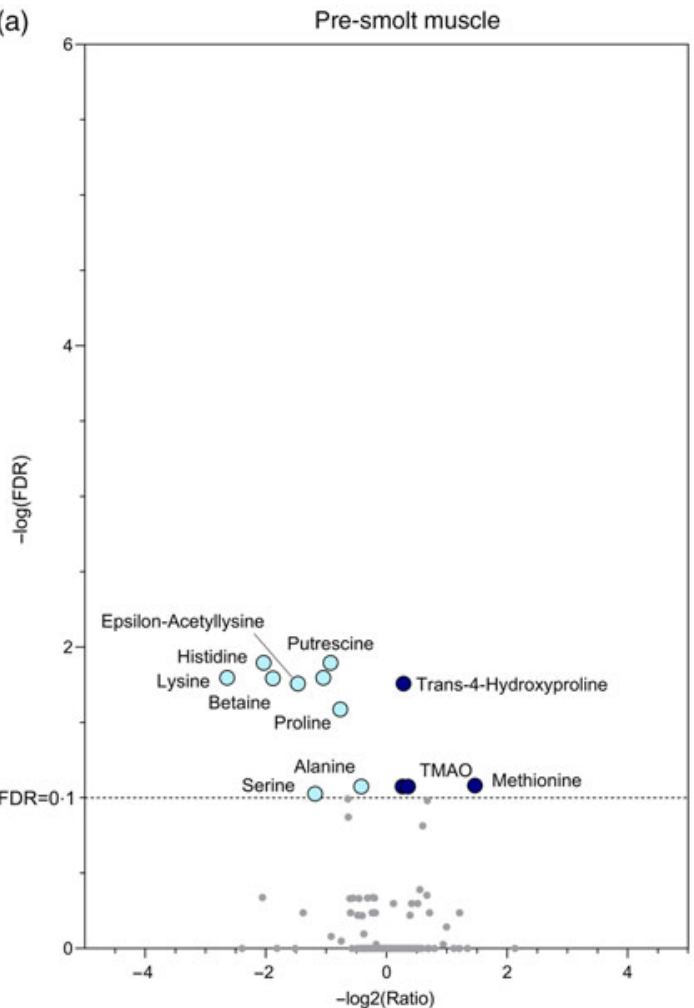

(b)

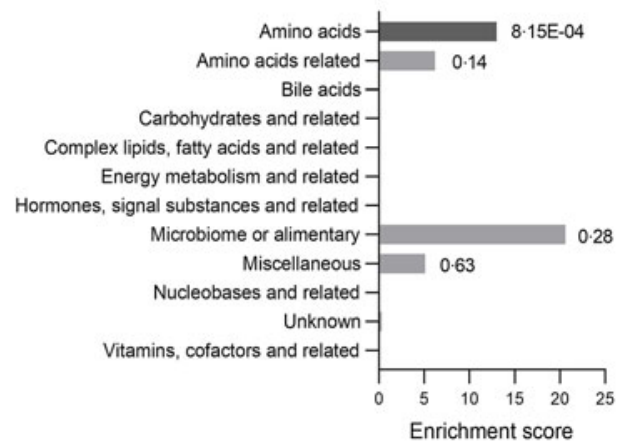

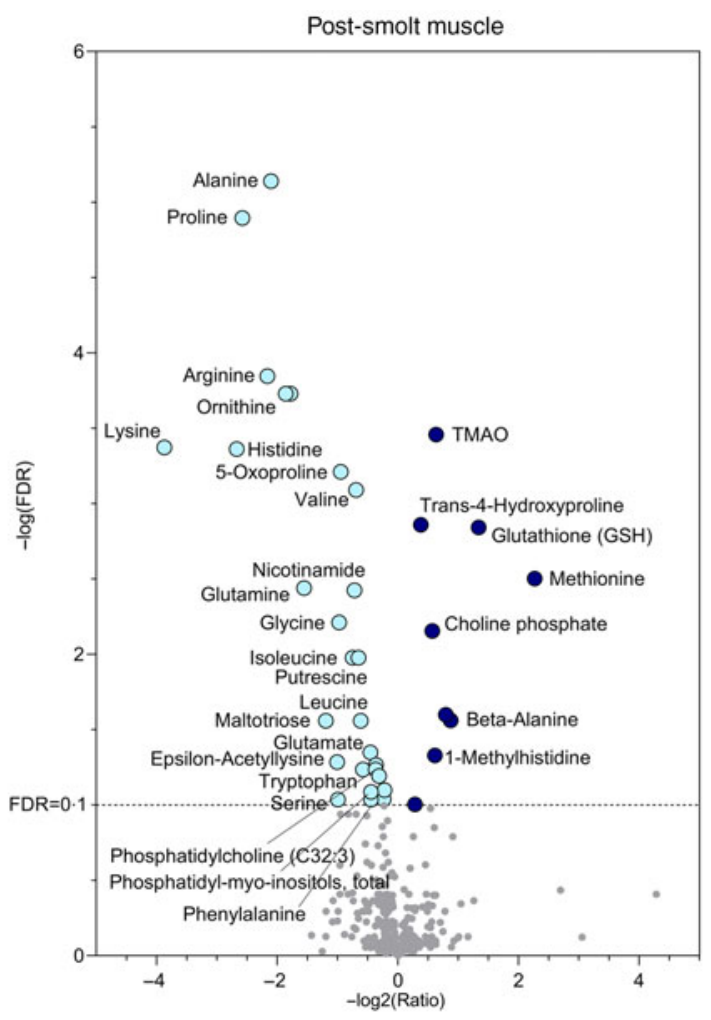

Post-smolt muscle

(c)

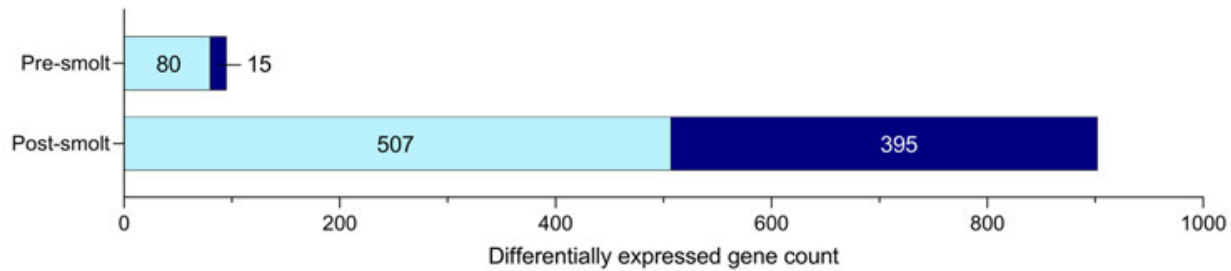

Fig. 2. Feeding a $1 \mathrm{C}$ nutrient surplus to Atlantic salmon during the smoltification period changed metabolic and transcriptomic profiles in muscle from both pre- and postsmolts. (a) Volcano plots showing metabolomic data from pre-smolt and post-smolt muscle. Differences in metabolites (dots) between the $1 \mathrm{C}+$ and Ctrl group were plotted as log2-transformed ratios of group means on the $x$-axis. Statistically different log2-ratios (FDR $<0 \cdot 1$, dashed line crossing the $y$-axis) were highlighted in shades of blue. In total, 13 and 37 out of 536 detected metabolites were different between $1 \mathrm{C}+$ and Ctrl profiles at pre- and post-smolt, respectively. Metabolites with negative log2-ratios indicate lower levels (light blue) in the $1 \mathrm{C}+$ compared with the Ctrl group, and vice versa for positive log2-ratios (dark blue). Metabolites with either high positive or negative log2-ratios display large magnitude differences between the groups. Metabolites-of-interest were labelled with their names (online Supplementary Table S4). (b) Metabolite class enrichment analysis shows that the largest differences between $1 \mathrm{C}+$ and Ctrl muscle were in the amino acids and their conjugates, which were particularly pronounced in post-smolt muscle. Calculated enrichment scores ( $\mathrm{x}$-axis) underlie a ratio of significant altered metabolites (FDR $<0.1)$ among detected ones in a metabolite class in relation to all significant altered metabolites among all detected ones in the global metabolic profiling. Significance of enrichment was assessed using Fisher's exact test with Benjamini and Hochberg correction ${ }^{(38)}$ and adjusted $P$-values given for each bar. Bold compound classes designate significant enrichment (adjusted $P$-value $<0.05$ ). (c) The total number of differentially expressed genes (DEG, RNA-seq) was higher in post-smolt (902) than in pre-smolt muscle (95) when using false-discovery adjusted $P$-value $<0.05$ and llog2-fold changel 1 as significance cut-offs. Numbers within bars represent DEG with either higher (upregulated) or lower mRNA levels (down-regulated) in $1 \mathrm{C}+$ muscle compared with Ctrl muscle. (a) $\bullet$, significantly higher metabolite level; $\mathrm{O}$, significantly lower metabolite level; $\bullet$, not significantly different; (c) $\square,+\log 2 \mathrm{FC} ; \square,-\log 2 \mathrm{FC}$. 


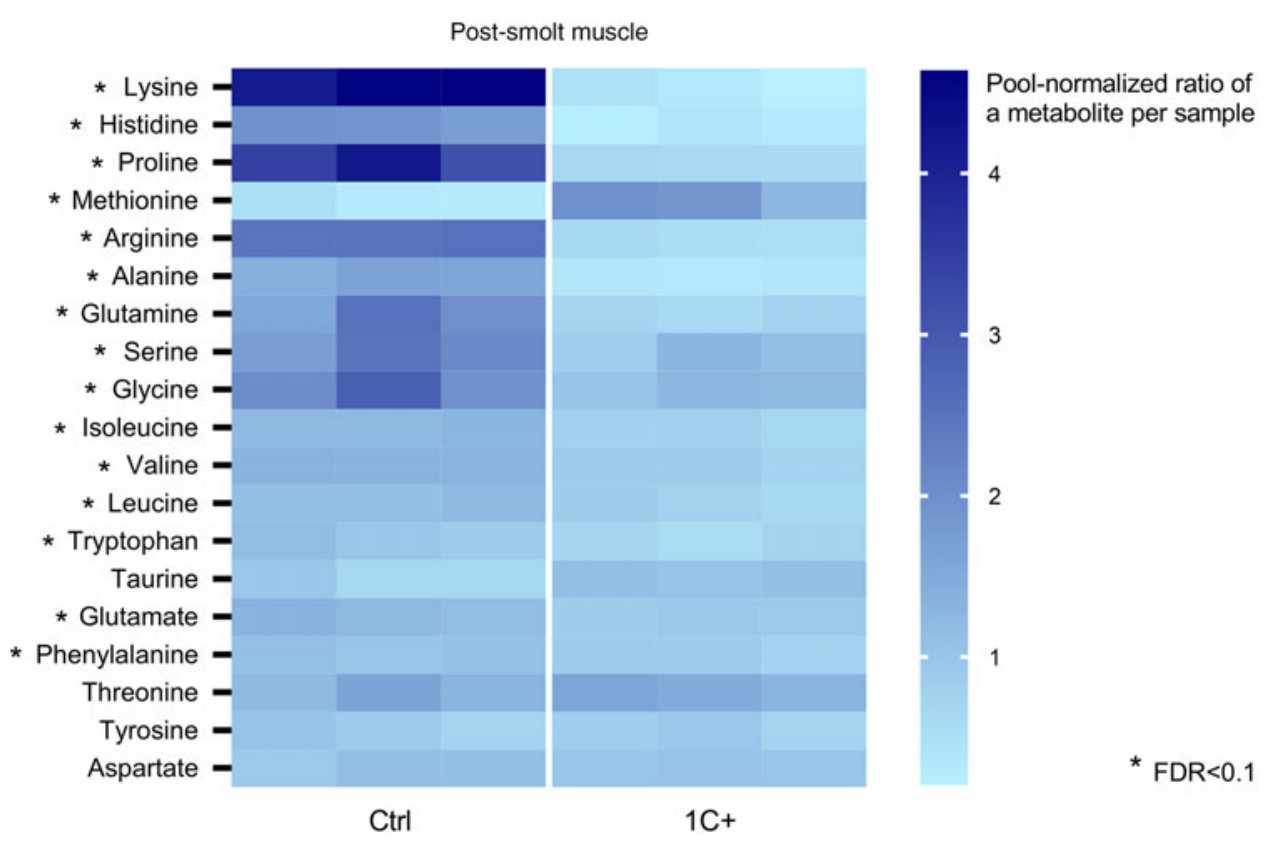

Fig. 3. Relative amino acid levels in muscle from $1 \mathrm{C}+$ and $\mathrm{Ctrl}$ fed salmon after smoltification (post-smolt). The heatmap illustrates individual sample levels of single amino acids and the $\mathrm{N}$-metabolite taurine that were detected in the global metabolic profiling. Cysteine and asparagine were not detected. Each cell illustrates the level of a single amino acid (rows) in each sample (columns) from each dietary group expressed as pool-normalised ratios in a colour scale. Asterisks $\left(^{*}\right)$ show significantly different amino acid levels between $1 \mathrm{C}+$ and $\mathrm{Ctrl}$ muscle (FDR $<0.1)$. Amino acids were sorted after the effect size (magnitude of log2ratio) between group means in the descending order. All metabolic data are provided in Supplementary Table S4.

Table S6). Samples were controlled for outliers and mRNA libraries showed no batch effects by sex, flow cell or tanks (online Supplementary Fig. S2). Gene expression profiles clustered by dietary treatment and particularly by life-stage of Atlantic salmon (online Supplementary Fig. S3). Hierarchical clustering of the samples shows a clear separation between the $1 \mathrm{C}+$ and Ctrl group at post-smolt (online Supplementary Fig. S3). More DEG between dietary groups were found in post-smolt (902 DEG) than in pre-smolt muscle (ninety-five DEG, Fig. 2(c)). The majority of the DEG show lower mRNA levels in 1C+ than in Ctrl for both pre- and post-smolt muscle (Fig. 2(c)). The annotated lists of DEG are given in online Supplementary Table S7 and volcano plots in online Supplementary Fig. S4 to visualise differential expression in pre- and post-smolt muscle.

\section{Metabolic characteristics of muscle from pre-smolt and post-smolt salmon}

The amino acids lysine, histidine and proline were the metabolites showing the biggest differences (highest pool-normalised ratio), whereas alanine, proline and arginine were the most significant metabolites (lowest adjusted $P$-value) between the dietary groups (Fig. 2(a) and 3, online Supplementary Table S4). Fourteen out of in total nineteen detected amino acids, including the $\mathrm{N}$-metabolite taurine, showed lower levels in 1C+ compared with Ctrl post-smolt muscle (Fig. 3). Lysine, histidine, alanine, proline, leucine, isoleucine, phenylalanine, tryptophan, serine, glycine, glutamine, arginine, valine and glutamate had significantly lower levels, while methionine had significantly higher levels in post-smolt muscle (Fig. 3 and online Supplementary Table S4).

Lysine, generally present in high proportions in fish muscle, is involved in growth and cross-linking of proteins such as collagen. Another major component of collagen is trans-4hydroxyproline, which was here elevated in 1C+ (Fig. 2(a)). Post-translational acetylation of lysine or lysine residues of histones and non-histone proteins forms derivatives like $\epsilon$-acetyllysine, which was decreased in $1 \mathrm{C}+.1 \mathrm{C}+$ comprised lower nicotinamide levels, which is an important epigenetic mediator of deacetylases and part of $\mathrm{NAD}^{+}$functioning in oxidationreduction reactions of intermediary metabolism. $1 \mathrm{C}+$ showed higher levels of $\beta$-alanine and the histidine conjugate 1-methylhistidine, which are together constituents of anserine important for muscle homoeostasis, $\mathrm{pH}$ buffering and antioxidant capacity. The amine oxide trimethylamine N-oxide, in short TMAO, was elevated in 1C+. Trimethylamine N-oxide is biosynthesised from choline and phosphatidylcholine and known to protect against the adverse effects of temperature, salinity, high urea and hydrostatic pressure. Choline was not different, but $1 \mathrm{C}+$ exhibited higher levels of choline phosphate (other name for phosphocholine), which is an intermediate in the synthesis of phosphatidylcholine. Phosphatidylcholine C32:3 was significantly lower in $1 \mathrm{C}+$. SAM acts as a methyl donor in the synthesis of phosphatidylcholine from phosphatidylethanolamine. Levels of phosphatidyl-myo-inositols and maltotriose were lower in $1 \mathrm{C}+$ than in Ctrl muscle.

$1 \mathrm{C}+$ muscle revealed lower levels of arginine, ornithine and putrescine, which is the direct precursor for polyamines 
(Fig. 2(a)). Polyamine synthesis is dependent on the availability of SAM, which was not detected in the metabolic profiling, but additional analysis of SAM revealed increased levels in 1C+ in post-smolt muscle (Fig. 4). SAH, synthesised upon donation of a methyl group from SAM, was not different between the dietary groups (online Supplementary Table S4 and Fig. 4). The ratio of SAM to SAH was higher in $1 \mathrm{C}+$ than in Ctrl. SAM synthesis depends on methionine, in which levels follow the dietary treatment with higher amounts in 1C+ than Ctrl (Fig. 2(a)). Methionine can get irreversible metabolised through transsulphuration into cysteine, taurine and the antioxidant GSH, which was significantly increased in 1C+ (Fig. 2(a)).

Pre-smolt muscle shares most of their changed metabolites with those that were different in post-smolt muscle (Fig. 2(a)). Common metabolites show the same direction of change between $1 \mathrm{C}+$ and Ctrl muscle. Methionine, trimethylamine $\mathrm{N}$-oxide and trans-4-hydroxyproline levels were increased, whereas levels of alanine, serine, putrescine, proline, histidine, lysine and $\epsilon$-acetyllysine were decreased in $1 \mathrm{C}+$ compared with the Ctrl (Fig. 2(a)). Betaine, an osmolyte and methyl group donor in the re-methylation of homocysteine to methionine, was decreased in pre-smolt $1 \mathrm{C}+$ but not in post-smolt (Fig. 2(a) and online Supplementary Table S4).

\section{Common differentially expressed genes between pre-smolt and post-smolt muscle}

Twenty-five DEG were shared between pre- and post-smolt muscle differences (online Supplementary Table S7). Among them (Table 2), decreased mRNA levels in $1 \mathrm{C}+$ muscle were found for two $S$-adenosylmethionine synthase paralogous genes (mat2a, mat2a-like), two genes encoding ferritin subunit paralogs (frim, frim-like) and four Na-coupled amino acid transporter paralogs (slc6a9-like, slc38a3-like, slc6a15-like, slc6a15-like). The gene encoding galactosidase $\alpha$ (gla), which is active in lysosomes and catalyses the removal of terminal $\alpha$-galactose groups from glycoproteins and glycolipids, was expressed at significantly lower levels in 1C+ muscle from both pre- and post-smolts. Three paralogs of the interferon-induced guanylatebinding protein 1 ( $g b p$-1-like) involved in endosomal trafficking,
Pre-smolt muscle

SAM

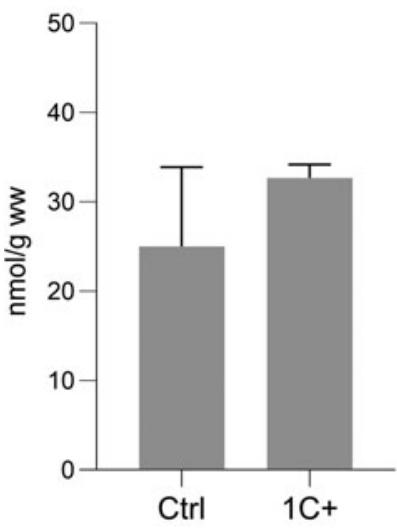

Post-smolt muscle
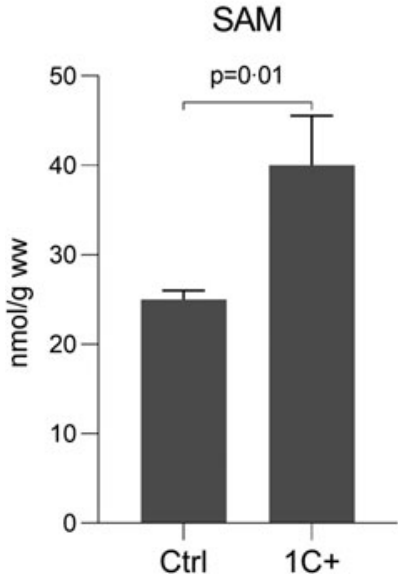

$\mathrm{SAH}$

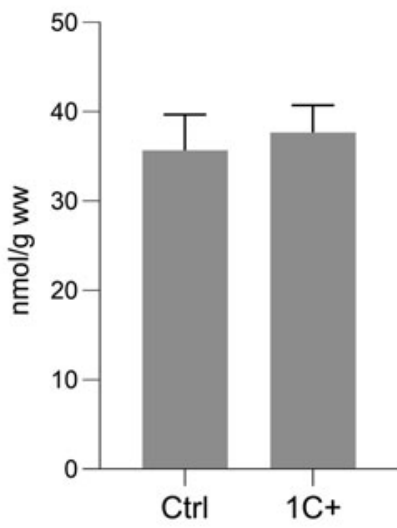

$\mathrm{SAH}$

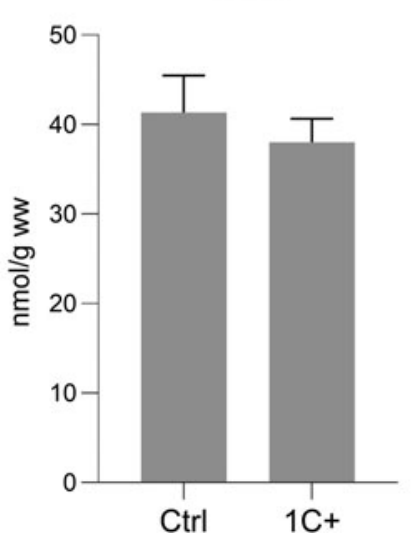

SAM/SAH ratio

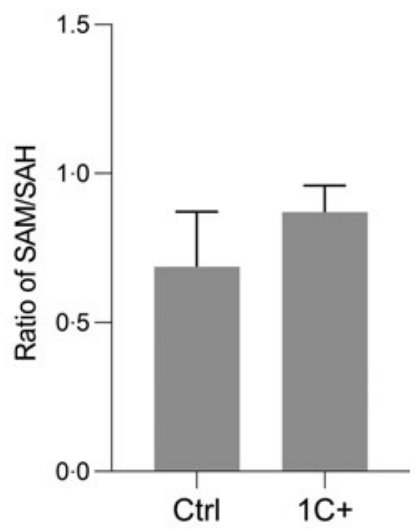

SAM/SAH ratio

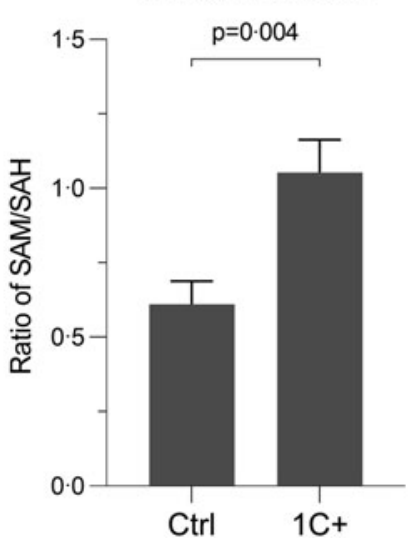

Fig. 4. S-adenosylmethionine (SAM) and S-adenosylhomocysteine (SAH) levels comparing $1 \mathrm{C}+$ and Ctrl in pre- and post-smolt salmon muscle assessed by HPLC. The ratio of SAM to SAH is given as a measure to study methylation capacity in the muscle. Statistical significance was assessed using a two-tailed $t$ test with $P<0.05$. $P$-values of significant differences were indicated above dark grey coloured bars. Non-significant differences were kept light grey. 
Table 2. Selected genes differentially expressed with diet in both pre-smolt and post-smolt muscle. List of all overlapping genes is provided in online Supplementary Table S7. Log2FC, log2-fold change. Adjusted $P$, adjusted $P$-value (output of DESeq2)

\begin{tabular}{|c|c|c|c|c|c|c|}
\hline \multirow[b]{2}{*}{ Abbreviation } & \multirow[b]{2}{*}{ Entrez ID } & \multirow[b]{2}{*}{ Gene description } & \multicolumn{2}{|c|}{ Pre-smolt muscle } & \multicolumn{2}{|c|}{ Post-smolt muscle } \\
\hline & & & Log2FC & Adjusted $P$ & Log2FC & Adjusted $P$ \\
\hline frim $^{*}$ & 106600949 & Ferritin, middle subunit & $-1 \cdot 6$ & $3.0 e-05$ & $-2 \cdot 28$ & $3.5 e-11$ \\
\hline frim-like* & 106600950 & Ferritin, middle subunit-like & -1.49 & $4 \cdot 8 e-03$ & $-2 \cdot 16$ & $2.0 e-06$ \\
\hline gla & 100380380 & Galactosidase $\alpha$ & -1.57 & $3.0 e-03$ & $-3 \cdot 68$ & $3 \cdot 4 e-61$ \\
\hline$g b p-1-l i k e^{*}$ & 106582240 & Interferon-induced guanylate-binding protein 1-like & $-2 \cdot 67$ & $1 \cdot 1 e-03$ & -1.41 & $1 \cdot 1 e-04$ \\
\hline$g b p-1-l i k e^{*}$ & 106582242 & Interferon-induced guanylate-binding protein 1-like & $-2 \cdot 45$ & $8 \cdot 6 e-03$ & $-1 \cdot 34$ & $4.4 e-05$ \\
\hline arhgap12-like* & 106566136 & Rho GTPase-activating protein 12-like & $-1 \cdot 1$ & $1.1 e-03$ & $-1 \cdot 34$ & $8 \cdot 3 e-07$ \\
\hline arhgap30-like* & 106605587 & Rho GTPase-activating protein 33-like & $-1 \cdot 06$ & $4.5 e-02$ & $1 \cdot 31$ & $3 \cdot 3 e-05$ \\
\hline mat2a* $^{*}$ & 106563191 & S-adenosylmethionine synthase isoform type-2 & -1.57 & $1 \cdot 3 e-15$ & -1.57 & $6 \cdot 3 e-25$ \\
\hline mat2a-like* & 106580346 & S-adenosylmethionine synthase isoform type-2-like & $-1 \cdot 64$ & $1.9 e-06$ & -1.99 & $6 \cdot 8 e-20$ \\
\hline slc6a9-like* & 106599379 & Sodium- and chloride-dependent glycine transporter 1-like & $-1 \cdot 19$ & $8 \cdot 0 e-03$ & $-1 \cdot 81$ & $3 \cdot 6 e-14$ \\
\hline slc38a3-like* & 106572384 & Sodium-coupled neutral amino acid transporter 3-like & -1.45 & $4 \cdot 7 e-02$ & $-3 \cdot 42$ & $1 \cdot 1 e-45$ \\
\hline slc6a15-like* & 106573109 & Sodium-dependent neutral amino acid transporter B(0)AT2-like & $-2 \cdot 33$ & $1.5 e-02$ & -4.54 & $2 \cdot 3 e-34$ \\
\hline slc6a15-like* & 106561596 & Sodium-dependent neutral amino acid transporter B(0)AT2-like & $-1 \cdot 8$ & $4 \cdot 8 e-02$ & $-5 \cdot 39$ & $7 \cdot 3 e-28$ \\
\hline
\end{tabular}

* Genes that received a human orthologue name when an official gene symbol was not available for the Atlantic salmon reference genome.

cytoskeleton regulation and autophagy were expressed at lower levels in 1C+. Two overlapping DEG encode Rho family GTPase-activating proteins (arbgap12-like, arbgap30-like) that function in a variety of cytoskeleton-dependent cell functions.

Lower expressed genes linked to mRNA translation and amino acid metabolisation in $1 \mathrm{C}+$ muscles from post-smolts

DEG between 1C+ and Ctrl (online Supplementary Table S7) have been associated with biological processes and molecular functions by ORA. ORA results from pre- and post-smolt muscles are listed separately in Supplementary Table S8 and S9, respectively. For post-smolt enrichment, solely down-regulated genes encoding amino acid-specific tRNA synthetases and ligases central in translation for protein synthesis account for the most enriched KEGG pathway, aminoacyl-tRNA biosynthesis (Fig. 5) and a few enriched gene ontology term categories listed in online Supplementary Table S9. Figure 5 shows also DEG enrichment in the KEGG pathway biosynthesis of amino acids entirely by lower expressed genes encoding a diverse group of enzymes driving amino acid metabolisation (Fig. 5, online Supplementary Table S9). Among them were genes like glna, cgl, shmt2, cbs-like, mat1a-like, mat2a-like, pycr1-like, p5cs-like, cps1, otc and $\arg 2$, which were listed in Table 3 and categorised after their functional roles or pathway affiliation. Concordant results were found with Gene Set Enrichment Analysis (online Supplementary Fig. S5) revealing aminoacyltRNA biosynthesis and biosynthesis of amino acids as the most down-regulated KEGG pathways in the post-smolt gene expression profiles.

Folate cycle key genes are expressed at lower levels in 1C+ muscle from post-smolts

Genes encoding mitochondrial homologs of folate cycle enzymes enriched the KEGG pathway one carbon pool by folate (Fig. 5, online Supplementary Table S9). Compared with the Ctrl, $1 \mathrm{C}+$ muscle showed lower expression of shmt2, mthfd1l, mthfd2-like, aldb1l2-like and mtdc-like (Table 3).
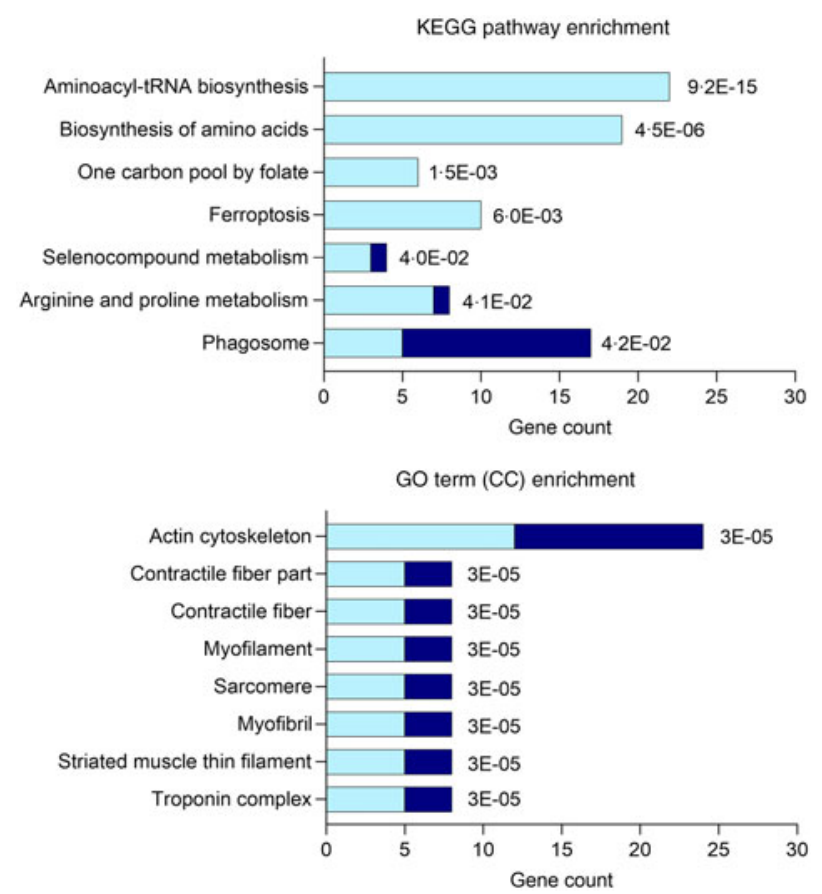

Fig. 5. Over-representation of differentially expressed genes (DEG) in KEGG pathways and cellular components (CC) between $1 \mathrm{C}+$ and Ctrl muscle after smoltification. Over-representation analysis (ORA) was performed to show functional enrichment of DEG. Bars illustrate the DEG number in a category. Shades of blue highlight DEG either with positive $(+\log 2 \mathrm{FC})$ or negative log2-fold changes (-log2FC). DEG with -log2FC show lower mRNA levels in $1 \mathrm{C}+$ than in Ctrl muscle. Adjusted $P$-values for each enrichment were associated with each category (bars). ORA is based on DEG with a llog2-fold changel $>1$ and adjusted $P$-value $<0.05$ when comparing gene expression of $1 \mathrm{C}+$ and Ctrl muscle. $\square+\log 2 \mathrm{FC} ; \square,-\log 2 \mathrm{FC}$.

\section{C+ muscle from post-smolts reveals lower gene expression in transmethylation and transsulphuration reactions}

Transmethylation reactions depend on the availability of SAM. peam3 (Table 3), encoding a N-methyltransferase that methylates phosphoethanolamine to phosphocholine using SAM as 
Table 3. Selected differentially expressed genes (DEG) between 1C+ and Ctrl post-smolt muscle categorised after their functional roles or pathway affiliation. Online supplementary Table S7 provides the full list of DEG between diet groups in post-smolts. Log2FC, log2-fold change. Adjusted $P$, adjusted $P$-value (output of DESeq2)

\begin{tabular}{|c|c|c|c|c|}
\hline Abbreviation & Entrez ID & Gene description & Log2FC & Adjusted $P$ \\
\hline \multicolumn{5}{|l|}{ Folate cycle } \\
\hline mthfd11 & 100306829 & Methylenetetrahydrofolate dehydrogenase (NADP + dependent) 1 like & -1.79 & $2 \cdot 7 \mathrm{e}-14$ \\
\hline shmt2 & 106566120 & Serine hydroxymethyltransferase 2 & -1.55 & $8 \cdot 8 \mathrm{e}-04$ \\
\hline mthfd2-like* & 106585270 & Bifunctional methylenetetrahydrofolate dehydrogenase/cyclohydrolase, mitochondrial-like & -1.51 & $1 \cdot 7 \mathrm{e}-25$ \\
\hline aldh1l2-like* & 106609325 & Mitochondrial 10-formyltetrahydrofolate dehydrogenase-like & $-1 \cdot 16$ & $9.9 \mathrm{e}-10$ \\
\hline aldh1l2-like* & 106576152 & Mitochondrial 10-formyltetrahydrofolate dehydrogenase-like & $-1 \cdot 15$ & $2 \cdot 8 \mathrm{e}-18$ \\
\hline$m t d c-l i k e^{*}$ & 106580352 & Bifunctional methylenetetrahydrofolate dehydrogenase/cyclohydrolase, mitochondrial-like & $-1 \cdot 1$ & $3 \cdot 9 e-21$ \\
\hline \multicolumn{5}{|c|}{ Methionine metabolism } \\
\hline mat1a-like* & 106613069 & S-adenosylmethionine synthase isoform type-1-like & $-5 \cdot 17$ & $7 \cdot 7 \mathrm{e}-09$ \\
\hline mat2a* & 106563191 & S-adenosylmethionine synthase isoform type-2 & -1.57 & $6 \cdot 3 e-25$ \\
\hline mat2a-like* & 106580346 & S-adenosylmethionine synthase isoform type-2-like & -1.99 & $6 \cdot 8 \mathrm{e}-20$ \\
\hline mat2a-like $^{*}$ & 106585265 & S-adenosylmethionine synthase isoform type-2-like & -1.24 & $1 \cdot 3 e-10$ \\
\hline \multicolumn{5}{|c|}{ Transsulphuration pathway } \\
\hline cbs-like ${ }^{*}$ & 106582148 & Cystathionine $\beta$-synthase-like & -1.21 & $4 \cdot 4 \mathrm{e}-03$ \\
\hline $\mathrm{cgl}$ & 100194964 & Cystathionine gamma-lyase & $-1 \cdot 74$ & $5.4 \mathrm{e}-44$ \\
\hline \multicolumn{5}{|c|}{ Transmethylation reactions } \\
\hline peam3 & 100380594 & Phosphoethanolamine N-methyltransferase 3 & -5.02 & $4 \cdot 7 \mathrm{e}-39$ \\
\hline \multicolumn{5}{|c|}{ Polyamine metabolism and ferroptosis } \\
\hline sat2 & 100380620 & Spermidine/spermine N1-acetyltransferase family member 2 & -1.27 & $2 \cdot 9 e-21$ \\
\hline sat2-like* & 106608759 & Diamine acetyltransferase 2 -like & $-2 \cdot 38$ & $2 \cdot 2 \mathrm{e}-07$ \\
\hline sat1-like* & 106563588 & Diamine acetyltransferase 1 -like & $-1 \cdot 74$ & $4 \cdot 5 e-20$ \\
\hline \multicolumn{5}{|c|}{ Arginine and proline metabolism } \\
\hline pycr1-like* & 106606436 & Pyrroline-5-carboxylate reductase 1 , mitochondrial-like & -1.34 & $1.0 \mathrm{e}-32$ \\
\hline glna & 100196183 & Glutamine synthetase & -2.93 & $2 \cdot 3 e-14$ \\
\hline p5cs-like & 106579029 & Delta-1-pyrroline-5-carboxylate synthase-like & $-2 \cdot 13$ & $8 \cdot 5 e-28$ \\
\hline p5cs-like* & 106589322 & Delta-1-pyrroline-5-carboxylate synthase-like & $-2 \cdot 35$ & $1 \cdot 7 \mathrm{e}-68$ \\
\hline cps 1 & 106586447 & Carbamoyl-phosphate synthase 1 & -1.79 & $1.9 \mathrm{e}-27$ \\
\hline sirt5-like* & 106560408 & NAD-dependent protein deacylase sirtuin-5, mitochondrial-like & -3.65 & $1.0 \mathrm{e}-03$ \\
\hline otc & 106586678 & Ornithine carbamoyltransferase & -1.94 & $8 \cdot 6 e-29$ \\
\hline $\arg 2$ & 106599105 & Arginase 2 & -1.33 & $1.0 \mathrm{e}-16$ \\
\hline \multicolumn{5}{|c|}{ Glutathione metabolisation and redox balance } \\
\hline gstp $1^{*}$ & 100136484 & Glutathione S-transferase P & -1.69 & $3 \cdot 1 \mathrm{e}-21$ \\
\hline chac1-like ${ }^{*}$ & 106611099 & Glutathione-specific gamma-glutamylcyclotransferase 1-like & -1.3 & $7 \cdot 8 \mathrm{e}-03$ \\
\hline mgst3-like & 106600407 & Microsomal glutathione S-transferase 3-like & $-2 \cdot 6$ & $7 \cdot 5 \mathrm{e}-03$ \\
\hline osgin1-like ${ }^{*}$ & 106565048 & Oxidative stress-induced growth inhibitor 2-like & -1.02 & $5 \cdot 7 e-07$ \\
\hline
\end{tabular}

* Genes that received a human orthologue name when an official gene symbol was not available for the Atlantic salmon reference genome.

substrate, was among the top DEG with the largest fold change showing lower mRNA levels in $1 \mathrm{C}+$ muscle. Table 3 also lists the transsulphuration pathway and vitamin $\mathrm{B}_{6}$-dependent genes $\mathrm{cgl}$ and cbs-like that were lower expressed in $1 \mathrm{C}+$. The transsulphuration pathway removes undesirable amounts of homocysteine through cystathionine and cysteine.

\section{C+ muscle from post-smolts shows lower expression} of genes linked to polyamine homoeostasis regulation

Polyamine homoeostasis is maintained by the spermidine/ spermine N1-acetyltransferase family member 1 and 2, also known as diamine acetyltransferases 1 and 2. sat2, sat2-like and its paralog sat1-like (Table 3) were lower expressed in $1 \mathrm{C}+$ than in the Ctrl muscle.

Ferroptosis and glutathione metabolising genes are expressed at lower levels in post-smolt 1C+ muscle

The ferroptosis KEGG pathway was enriched by solely lower expressed genes in both pre-smolt and post-smolt muscle comparisons (Fig. 5, online Supplementary Table S8). Decreased expression in 1C+ was found for ferritin middle subunit paralogs (frim, frim-like), ferritin heavy polypeptide
1-1 (frib), sat2, sat2-like, sat1-like and slc40a1 (online Supplementary Table S9). One regulatory pathway of ferroptosis is the metabolism of GSH. 1C+ showed decreased expression of the GSH regulating and conjugating enzymes encoded by gstp1, chac1-like, mgst3-like and osgin1-like (Table 3).

\section{Differential expression of myofibrillar proteins and genes regulating cytoskeletal organisation in post-smolt muscle}

Actin cytoskeleton, striated muscle thin filament and the troponin complex were among the DEG-enriched cellular component ontology terms (Fig. 5). Several genes encoding various proteins building the actin cytoskeleton such as myosin and troponins I and $\mathrm{T}$ were differentially expressed between $1 \mathrm{C}+$ and $\mathrm{Ctrl}$ muscle (online Supplementary Table S9). Transgelin-3 is an actin-binding protein of the cytoskeleton, and differences in tagln-3-like expression showed the highest fold change in the post-smolt data, where it was expressed in Ctrl, but not expressed in 1C+ muscle (Fig. 6(a)). Contrarily, 1C+ muscle revealed an increased expression of myoz2 encoding an $\alpha$-actinin- and $\gamma$-filamin-binding $Z$ line protein involved in myofibrillogenesis. Fibrillar proteins and extracellular matrix regulating proteins involved in homoeostasis and organisation 
(a)
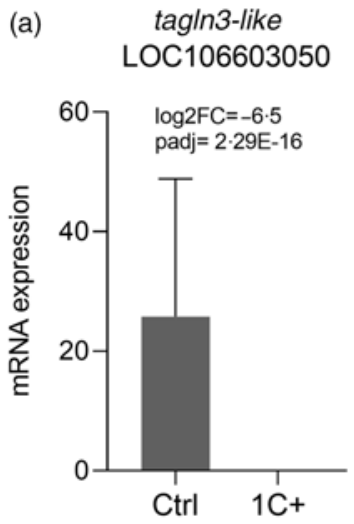

(b)

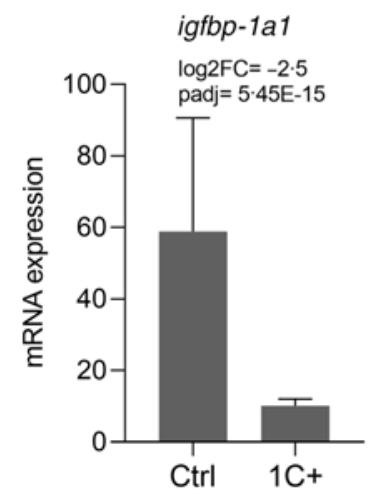

myoz2

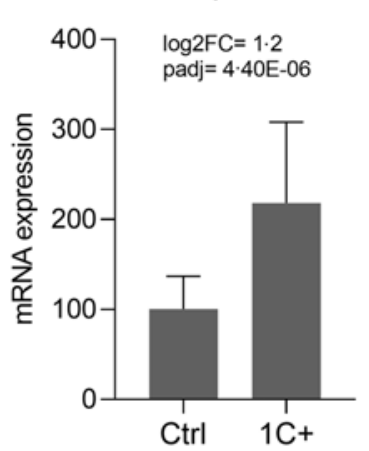

egr1

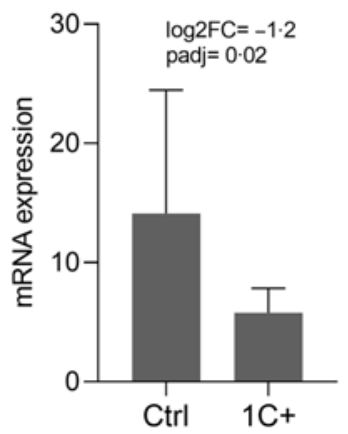

mmp 13

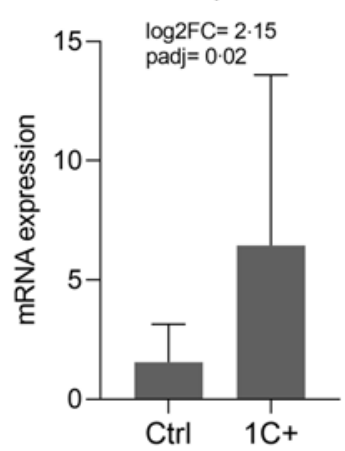

mrf4

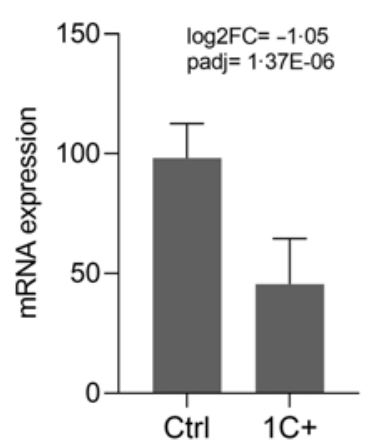

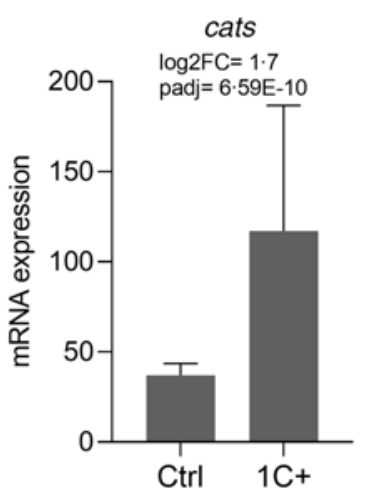

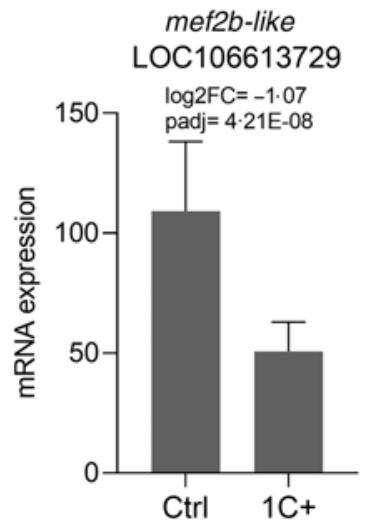

Fig. 6. mRNA expression of selected differentially expressed genes (DEG) that encode myofibrillar proteins and proteins regulating cytoskeletal organisation (a), and genes associated with growth and myogenesis regulating factors (b) between $1 \mathrm{C}+$ and Ctrl muscle from post-smolts. Bar graphs show the group mean ( $n$ 9) of normalised mRNA read counts detected by RNA-seq. (a) tagln3-like: transgelin-3-like, myoz2: myozenin 2, mmp13: collagenase 3, cats: cathepsin S; (b) igfbp1a1: insulin-like growth factor binding protein 1 paralog A1, egr1: early growth response 1, mrf4: myogenic regulatory factor 4 , mef2b-like: myocyte-specific enhancer factor 2B-like. Log2FC: log2-fold change; padj: adjusted $P$-value (DESeq2 output).

of the sarcomeric cytoskeleton were more highly expressed in $1 \mathrm{C}+$ than in Ctrl muscle. Among them, there were DEG encoding various collagen $\alpha$ paralogs, collagenase 3 and cathepsin $\mathrm{S}$ (Fig. 6(a) and online Supplementary Table S7). A few other DEG encoding muscular cytoskeleton assembly proteins such as integrin $\alpha$ and $\beta$ subunits (itgae, itb2, LOC106586548, LOC106602819) and tubulin $\alpha$ chain-like (LOC106565439) showed increased expression in 1C+ (online Supplementary Table S7).

\section{Differential expression of factors mediating growth and myogenesis in post-smolt muscle}

Expression of igfbp-1a1 encoding a protein that inhibits or potentiates IGF-1 growth promoting functions was decreased in 1C+ muscle (Fig. 6(b)). The early growth response 1 encoding gene (egr1) encodes a transcription factor that can be induced by growth factors in the IGF- 1 cascade and that acts in a wide range of growth and cell differentiation-related processes. egr1 showed decreased expression in 1C+ muscle. Maintenance of muscle fibre differentiation is regulated by myogenic regulatory factor 4 encoded by $m r f 4$ that was lower expressed in 1C+ (Fig. 6(b)). Lower expression was also found for mef2b-like that encodes a paralog of myocyte-specific enhancer factor 2B, a transcriptional activator of growth-related genes (Fig. 6(b)).

\section{Discussion}

A moderate surplus of dietary methionine, folic acid, vitamin $\mathrm{B}_{6}$ and $\mathrm{B}_{12}$ above the current requirements and recommended levels for Atlantic salmon given with the Med-1C feed improved growth, that is, increased body weight in the on-growing saltwater period as recently described by Espe et al. ${ }^{(7)}$. We aimed to understand how salmon fed the Med-1C feed, which is identical to the $1 \mathrm{C}+$ feed in the present study improved growth during the on-growing saltwater period ${ }^{(7)}$. We investigated metabolic and molecular signatures of muscle from the same fish as in the previous study ${ }^{(7)}$ from the Ctrl (Low-1C) and $1 \mathrm{C}+$ (Med-1C) group before and after smoltification to acquire deeper insight in metabolic properties, pathways and possible nutrient-gene interactions involved in the observed difference in growth.

We found a higher number of different metabolites and DEG in muscle of salmon from the on-growing saltwater period (post-smolt) than in the fresh water period (pre-smolt). That is in line with the phenotype earlier described for the same 
experimental fish between the two dietary groups after smoltification $^{(7)}$. Espe et al. reported higher protein accretion (deposition of muscle protein) and higher retention of indispensable amino acids in post-smolts compared with pre-smolts ${ }^{(7)}$. Moreover, the retention of methionine was higher when dietary inclusion was lowest while none of the other indispensable amino acid levels differed between the two feeds ${ }^{(7)}$. Results from the present study were in line with Espe et al. showing lower methionine levels in Ctrl muscle when dietary inclusion was lower. Free amino acids in Ctrl muscle from the present study were increased, which confirms the results from Espe et al. showing that utilisation or deposition of amino acids was lower when the Ctrl diet was fed to salmon. This points to free amino acids accumulation as an indicator of a reduced growth capacity in both salmon and zebrafish ${ }^{(7,16,42)}$. Conformably, increased mRNA expression of genes involved in translation and amino acid metabolisation suggests a readiness for translation with a following mobilisation of amino acids for protein synthesis in Ctrl muscle. Amino acids have the ability to influence gene expression through modulating the initiation phase of mRNA translation, but the likelihood that an aminoacyl-tRNA synthetase acts in translation for protein synthesis may also depend on other transcription factors that can explain the reduced growth capacity in the Ctrl group ${ }^{(43,44)}$.

Since the demand of methionine is shared between protein synthesis and transmethylation reactions, efficient metabolisation of methionine requires both dietary methionine and B vitamin supply in the 1C metabolism, and the endogenous supply of betaine from choline. For $1 \mathrm{C}+$ muscle, the demand of methionine for protein growth was most likely met through dietary and endogenous supply of methionine and the $\mathrm{B}$ vitamins. Contrarily, Ctrl muscle showed increased expression of key genes in the mitochondrial folate cycle to meet the demand for methionine in protein synthesis and other reactions. Espe et al. reported lower cystathionine levels in Ctrl muscle, which indicates that the dietary levels of B vitamins were not sufficient $^{(7)}$. When methionine gets less incorporated into protein, it will be metabolised through SAM to a variety of other metabolites. In the present study, 1C+ muscle showed increased levels of both SAM, the mediator of transmethylation reactions and GSH synthetised through the transsulphuration pathway. Ctrl muscle revealed increased expression of S-adenosylmethionine synthase isoforms and vitamin $\mathrm{B}_{6}$-dependent enzymes suggesting stronger regulation of methionine metabolisation, transsulphuration and folate cycle in the $1 \mathrm{C}$ metabolism. Further, peam 3 expression was increased suggesting increased synthesis of phosphatidylcholine from phosphoethanolamine; however, we found only one phosphatidylcholine (C32:3) significantly increased in the Ctrl group. Except for increased phosphatidylcholine and decreased choline-phosphate levels in Ctrl muscle, we hardly found an effect on lipid or fatty acid composition in muscle that is in line with the previous results from the same experimental fish $^{(7)}$. Overall, metabolic and RNA-seq results from muscle in this study show that the demand for dietary folic acid and methionine seems larger in Ctrl fed salmon to counterbalance the need for protein synthesis and growthrelated investments, which was more pronounced in the saltwater period. However, we cannot be certain whether the amounts of all $1 \mathrm{C}$ nutrients together or solely one of them were the main driving factors that improved growth when fed the $1 \mathrm{C}+$ feed consisting of a combined surplus package of methionine, folic acid, vitamin $\mathrm{B}_{6}$ and $\mathrm{B}_{12}$.

Skeletal muscle growth in fish is plastic and involves the enlargement of already existing muscle fibres (hypertrophy) and the recruitment of new fibres (hyperplasia). The balance between both is dependent on genetic differences and extrinsic factors such as early nutritional history ${ }^{(31,45)}$. It has been suggested that increased muscle hypertrophic growth in salmon is also associated with the stimulation of protein synthesis by diet balanced in micronutrients, particularly in B vitamins ${ }^{(5)}$. In general, fish growth and muscle maturation are centrally regulated through GH acting on receptors on the sarcolemma that indirectly initiates IGFBP production by IGF from the liver and by myogenic regulatory factors, respectively ${ }^{(20,21,26)}$. We found significantly higher mRNA expression of genes encoding factors involved in growth and transcription, which suggests stronger stimulation of myogenesis and growth in the Ctrl than in the $1 \mathrm{C}+$ group. Other genes that encode GHR1 and GHR2 precursors, IGF-1 and a few more IGFBP had higher mRNA levels in Ctrl muscle but were either below the chosen threshold | $\log 2 \mathrm{FC} \mid<1$ or were not significant differentially expressed. Espe et al. reported from targeted RT-qPCR analysis in muscle that $g h r$ was significantly increased, whereas igf-1 and its receptor were not different but showed the same trend with higher expression in Ctrl muscle ${ }^{(7)}$. Mechanisms explaining the increased expression of growth-stimulating factors in Ctrl muscle and the lower growth reported for this group are indicative of compensatory growth regulation at local tissue level. A compensatory regulation of systemic and local components of the GH/IGF axis has been shown for gilthead sea bream (Sparus aurata) fed diets highly replaced marine ingredients by alternative raw materials ${ }^{(46)}$. The ability of increased free amino acids in muscle to stimulate translation and protein accretion on the one hand, and a likely higher methionine, SAM, GSH and B vitamin demand on the other hand, may account as factors for the reduced growth capacity in the Ctrl group ${ }^{(44)}$.

Muscle tissue consists of muscle fibres and myofibrils with a complex and highly organised network, the sarcomeric cytoskeleton that interlinks filaments with contractile, structural and regulatory proteins ${ }^{(20)}$. RNA-seq analysis revealed differences in expression of genes encoding a wide range of actin-binding and structural proteins such as troponin and myosin, but also proteins regulating cytoskeletal re-organisation. Interestingly, in case of transgelin-3, which is involved in membrane trafficking and actin-binding, we could not detect any mRNAs in $1 \mathrm{C}+$, but its transcripts were present in Ctrl muscle. Tissue remodelling involves both protein synthesis and degradation through proteolytic activity that are central to nutrient supply adaptation and homoeostasis of a tissue ${ }^{(20,47)}$. Here, we found increased expression of collagenase 3 and cathepsin $\mathrm{S}$ in $1 \mathrm{C}+$ muscle that suggest increased proteolysis capacity thus tissue remodelling. The expression of proteolytic enzymes has also been associated with quality measures such as high firmness of salmon filet ${ }^{(48)}$. Overall, RNA-seq results from 1C+ post-smolts imply increased muscle tissue remodelling, but less stimulation of differentiation and growth in muscle. However, analysis of muscle cellularity is 
needed to provide more details on structural changes and possible quality measures of salmon muscle.

Ctrl muscle from the present study showed increased levels of the polyamine precursor putrescine and increased expression of ferritin subunits and enzymes regulating polyamine levels. Activation of sat1 has been linked to lipid peroxidation and ferroptosis stimulation upon reactive oxygen species (ROS) formation in cell culture ${ }^{(49)}$. The metabolism of amino acids is tightly linked to the regulation of ferroptosis, which is an irondependent form of cell death ${ }^{(50,51)}$. Ferroptosis is characterised by the accumulation of intracellular lipid $\operatorname{ROS}^{(51)}$ upon intracellular iron accumulation. Under oxidative stress conditions, methionine can be converted through the transsulphuration pathway to GSH that further exerts antioxidant effects ${ }^{(52)}$. Ferritin contributes to decrease in oxidative stress besides main functions in intracellular iron storage ${ }^{(50,53,54)}$. This might explain the increased mRNA levels of ferritin and the depletion of GSH that suggest higher GSH utilisation with increased oxidative load in the Ctrl muscle, possibly associated with increased polyamine metabolism $^{(13,49,51,55)}$. Polyamines, produced from putrescine and ornithine, regulate fundamental cellular processes such as cell growth and proliferation but can also affect redox balance as polyamine acetylation (degradation) can promote ROS affecting the oxidative load ${ }^{(56)}$. However, central enzymes in polyamine synthesis were not differentially expressed, whereas sat 2 and sat1-like expression was increased, suggesting higher degradation to maintain polyamine homoeostasis thus affecting redox balance in Ctrl muscle ${ }^{(13,56)}$. Interestingly, Ctrl muscle showed decreased expression of glutathione S-transferase P that suggests GSH depletion through S-glutathionylation of functional and structural proteins to maintain cellular redox homoeostasis in response to ROS in the Ctrl group ${ }^{52,57)}$.

Adequate amounts of micronutrients prior to biological transformations such as smoltification in salmonids can be a decisive factor for later growth. Feeding a 1C-nutrient surplus prior to smoltification affected growth of pre-smolts marginally but that of post-smolts significantly, which indicates metabolic, possibly epigenetic programming in muscle. Changes in methionine levels can directly impact methyl-donor availability with possible consequences for DNA and histone tail methylation ${ }^{(58-60)}$. Differences we found in 6 -acetyllysine amounts and sirt5-like mRNA expression in muscle point to effects on histone tail or non-histone protein acetylation, but also solely to non-enzymatic acetylation of free lysine ${ }^{(61-63)}$. One can speculate whether a dietary $1 \mathrm{C}$-nutrient surplus as for the $1 \mathrm{C}+$ feed can introduce life-long changes in epigenetic profiles that affect muscle gene expression and improve growth ${ }^{(64,65)}$. Future studies should consider integration analysis of DNA methylation, histone methylation and acetylation marks to provide a deeper insight into nutrient-sensitive regulation of epigenetic mechanisms involved in growth, and whether post-smolt growth can be programmed prior to smoltification or during early development ${ }^{(12,66-71)}$.

\section{Conclusion}

A dietary surplus of methionine, folate, vitamin $\mathrm{B}_{6}$ and $\mathrm{B}_{12}$ above current requirement and recommended levels throughout smoltification appears to be beneficial in protein accretion for improved growth in farmed Atlantic salmon. The results from this study support that the availability of methionine along with $\mathrm{B}$ vitamins is important to efficiently use amino acids for protein growth. The metabolic signatures point to improved growth and redox capacity through improved utilisation and metabolisation of amino acids in muscle of $1 \mathrm{C}+$ fed salmon. We have also demonstrated that transcriptional changes in muscle in response to a dietary 1C-nutrient surplus relate to translation, mobilisation of amino acids, cytoskeletal organisation and growth. Understanding how growth is controlled by non-genetic mechanisms becomes important for a rapidly growing aquaculture industry whose concerns are to optimise production, sustainability and quality.

\section{Acknowledgements}

The authors thank the provision of the Atlantic salmon muscle through the project NutrEpi (NFR grant number 267787), and Tårn Helgøy Thomsen and staff at Skretting ARC (Stavanger, Norway) for performing the feeding trial and providing the feed. The authors are grateful to Jorge M.O. Fernandes's laboratory at Nord University (Bodø, Norway), particularly Qirui Zhang and Martina E.L. Kopp for assisting the high-throughput sequencing. The authors thank Metanomics Health GmbH (Berlin, Germany) for $\mathrm{MxP}^{\circledR}$ Global Profiling. The authors also would thank Eva Mykkeltvedt at IMR for technical assistance in sampling. Graphical abstract was created with BioRender.com.

This work was supported by the Research Council of Norway through the grant 295118 and by the Institute of Marine Research (Bergen, Norway). The Research Council of Norway had no role in the design, analysis or writing of this article.

A. A., M. E. and K. H. S conceived and designed the research. $\mathrm{V}$. V. provided the feed and performed the feeding trial. A. A. and J. M. O. F. prepared the libraries, and P. W. and T. S. performed bioinformatics analysis for the RNA-seq results. A. A., K. H. S., M. E. and T. S. analysed and interpreted data. A. A. drafted the manuscript. All authors read and approved the final manuscript.

The authors have no financial or personal conflicts of interest to declare.

\section{Supplementary material}

For supplementary materials referred to in this article, please visit https://doi.org/10.6084/m9.figshare.14484489.v1 and https:// doi.org/10.1017/S0007114521002336

\section{References}

1. Aas TS, Ytrestoyl T \& Asgard T (2019) Utilization of feed resources in the production of Atlantic salmon (Salmo salar) in Norway: an update for 2016. Aquacult Rep 15, 100216.

2. Hamre K, Sissener NH, Lock EJ, et al. (2016) Antioxidant nutrition in Atlantic salmon (Salmo salar) parr and post-smolt, fed diets with high inclusion of plant ingredients and graded levels of micronutrients and selected amino acids. PeerJ 4, e2688.

3. Hemre GI, Lock EJ, Olsvik PA, et al. (2016) Atlantic salmon (Salmo salar) require increased dietary levels of B-vitamins 
when fed diets with high inclusion of plant based ingredients. PeerJ 4, e2493.

4. Taylor JF, Vera LM, De Santis C, et al. (2019) The effect of micronutrient supplementation on growth and hepatic metabolism in diploid and triploid Atlantic salmon (Salmo salar) parr fed a low marine ingredient diet. Comp Biochem Physiol B Biochem Mol Biol 227, 106-121.

5. Hamre K, Bjornevik M, Espe M, et al. (2020) Dietary micronutrient composition affects fillet texture and muscle cell size in Atlantic salmon (Salmo salar). Aquacult Nutr 26, 936-945.

6. Vera LM, Hamre K, Espe M, et al. (2020) Higher dietary micronutrients are required to maintain optimal performance of Atlantic salmon (Salmo salar) fed a high plant material diet during the full production cycle. Aquaculture 528, 735551.

7. Espe M, Vikesa V, Thomsen TH, et al. (2020) Atlantic salmon fed a nutrient package of surplus methionine, vitamin $\mathrm{B}_{12}$, folic acid and vitamin $\mathrm{B}_{6}$ improved growth and reduced the relative liver size, but when in excess growth reduced. Aquacult Nutr 26, 477-489.

8. Espe M, Skjaerven KH, Chen M, et al. (2020) The level of 1C diets fed prior to cell isolation affects lipid metabolism in primary liver cells isolated from Atlantic salmon (Salmo salar). Aquacult Nutr 26, 1019-1025.

9. NRC (2011) Nutrient Requirements of Fish and Shrimp. Washington, DC: The National Academies Press.

10. Saito T, Whatmore P, Taylor JF, et al. (2020) Micronutrient supplementation affects transcriptional and epigenetic regulation of lipid metabolism in a dose-dependent manner. Epigenetics-Us, 1-18.

11. Ducker GS \& Rabinowitz JD (2017) One-Carbon metabolism in health and disease. Cell Metab 25, 27-42.

12. Clare CE, Brassington AH, Kwong WY, et al. (2019) One-Carbon metabolism: linking nutritional biochemistry to epigenetic programming of long-term development. Annu Rev Anim Biosci 7, 263-287.

13. Pegg AE (2008) Spermidine/spermine-N-1-acetyltransferase: a key metabolic regulator. Am J Physiol-Endoc $M$ 294, E995-E1010.

14. Ouyang Y, Wu Q, Li JJ, et al. (2020) S-adenosylmethionine: a metabolite critical to the regulation of autophagy. Cell Proliferat 53, e12891.

15. Francioso A, Conrado AB, Mosca L, et al. (2020) Chemistry and biochemistry of sulfur natural compounds: key intermediates of metabolism and redox biology. Oxid Med Cell Longev 2020 , 8294158.

16. Skjaerven KH, Jakt LM, Dahl JA, et al. (2016) Parental vitamin deficiency affects the embryonic gene expression of immune-, lipid transport- and apolipoprotein genes. Sci Rep 6, 34535 .

17. Skjaerven KH, Jakt LM, Fernandes JMO, et al. (2018) Parental micronutrient deficiency distorts liver DNA methylation and expression of lipid genes associated with a fatty-liver-like phenotype in offspring. Sci Rep $\mathbf{8}, 3055$.

18. Bower NI, Li XJ, Taylor R, et al. (2008) Switching to fast growth the insulin-like growth factor (IGF) system in skeletal muscle of Atlantic salmon. J Exp Biol 211, 3859-3870.

19. Velloso CP (2008) Regulation of muscle mass by growth hormone and IGF-I. Brit J Pharmacol 154, 557-568.

20. Johnston IA, Bower NI \& Macqueen DJ (2011) Growth and the regulation of myotomal muscle mass in teleost fish. J Exp Biol 214, 1617-1628.

21. Fuentes EN, Valdes JA, Molina A, et al. (2013) Regulation of skeletal muscle growth in fish by the growth hormone Insulin-like growth factor system. Gen Comp Endocr 192, 136-148.
22. Valente LMP, Moutou KA, Conceicao LEC, et al. (2013) What determines growth potential and juvenile quality of farmed fish species? Rev Aquacult 5, S168-S193.

23. Reindl KM \& Sheridan MA (2012) Peripheral regulation of the growth hormone-insulin-like growth factor system in fish and other vertebrates. Comp Biochem Phys A 163, 231-245.

24. Johnston IA (2006) Environment and plasticity of myogenesis in teleost fish. J Exp Biol 209, 2249-2264.

25. Le Grand F \& Rudnicki MA (2007) Skeletal muscle satellite cells and adult myogenesis. Curr Opin Cell Biol 19, 628-633.

26. Perez-Sanchez J, Simo-Mirabet P, Naya-Catala F, et al. (2018) Somatotropic axis regulation unravels the differential effects of nutritional and environmental factors in growth performance of marine farmed fishes. Front Endocrinol 9, 687.

27. Rolland M, Dalsgaard J, Holm J, et al. (2015) Dietary methionine level affects growth performance and hepatic gene expression of GH-IGF system and protein turnover regulators in rainbow trout (Oncorbynchus mykiss) fed plant protein-based diets. Comp Biochem Physiol B: Biochem Mol Biol 181, 33-41.

28. Hevroy EM, El-Mowafi A, Taylor RG, et al. (2007) Lysine intake affects gene expression of anabolic hormones in Atlantic salmon, Salmo salar. Gen Comp Endocrinol 152, 39-46.

29. Johnston IA, Manthri S, Smart A, et al. (2003) Plasticity of muscle fibre number in seawater stages of Atlantic salmon in response to photoperiod manipulation. J Exp Biol 206, 3425-3435.

30. Johnston IA, Manthri S, Alderson R, et al. (2003) Freshwater environment affects growth rate and muscle fibre recruitment in seawater stages of Atlantic salmon (Salmo salar L.). J Exp Biol 206, 1337-1351.

31. Bjornevik M, Beattie C, Hansen T, et al. (2003) Muscle growth in juvenile Atlantic salmon as influenced by temperature in the egg and yolk sac stages and diet protein level. J Fish Biol 62 , 1159-1175.

32. Campos C, Valente LMP, Conceicao LEC, et al. (2013) Temperature affects methylation of the myogenin putative promoter, its expression and muscle cellularity in Senegalese sole larvae. Epigenetics-Us 8, 389-397.

33. Amaral IPG \& Johnston IA (2012) Circadian expression of clock and putative clock-controlled genes in skeletal muscle of the zebrafish. Am J Physiol-Reg I 302, R193-R206.

34. Bower NI \& Johnston IA (2010) Discovery and characterization of nutritionally regulated genes associated with muscle growth in Atlantic salmon. Physiol Genomics 42a, 114-130.

35. Espe M, Hevroy EM, Liaset B, et al. (2008) Methionine intake affect hepatic sulphur metabolism in Atlantic salmon, Salmo salar. Aquaculture 274, 132-141.

36. Espe M, Andersen SM, Holen E, et al. (2014) Methionine deficiency does not increase polyamine turnover through depletion of hepatic S-adenosylmethionine in juvenile Atlantic salmon. Br J Nutr 112, 1274-1285.

37. Wang W, Kramer PM, Yang S, et al. (2001) Reversed-phase high-performance liquid chromatography procedure for the simultaneous determination of S-adenosyl-L-methionine and S-adenosyl-L-homocysteine in mouse liver and the effect of methionine on their concentrations. J Chromatogr B Biomed Sci Appl 762, 59-65.

38. Benjamini Y \& Hochberg Y (1995) Controlling the false discovery rate - a practical and powerful approach to multiple testing. $J$ R Stat Soc B 57, 289-300.

39. Love MI, Huber W \& Anders S (2014) Moderated estimation of fold change and dispersion for RNA-seq data with DESeq2. Genome Biol 15, 1-21.

40. Yu G, Wang LG, Han Y, et al. (2012) ClusterProfiler: an R package for comparing biological themes among gene clusters. OMICS 16, 284-287. 
41. Subramanian A, Tamayo P, Mootha VK, et al. (2005) Gene set enrichment analysis: a knowledge-based approach for interpreting genome-wide expression profiles. Proc Natl Acad Sci USA 102, 15545-15550.

42. Skjærven KH, Oveland E, Mommens M, et al. (2020) Out-of-season spawning affects the nutritional status and gene expression in both Atlantic salmon female broodstock and their offspring. Comp Biochem Physiol A Mol Integr Physiol 247, 110717.

43. Guo M \& Schimmel P (2013) Essential nontranslational functions of tRNA synthetases. Nat Chem Biol 9, 145-153.

44. Kimball SR \& Jefferson LS (2004) Amino acids as regulators of gene expression. Nutr Metab 1, 3

45. Morkore T, Ruohonen K \& Kiessling A (2009) Variation in texture of farmed Atlantic salmon (Salmo salar 1). relevance of muscle fiber cross-sectional area. J Texture Stud $\mathbf{4 0}$, $1-15$.

46. Benedito-Palos L, Saera-Vila A, Calduch-Giner JA, et al. (2007) Combined replacement of fish meal and oil in practical diets for fast growing juveniles of gilthead sea bream (Sparus aurata L.) Networking of systemic and local components of GH/IGF axis. Aquaculture 267, 199-212.

47. Lavajoo F, Perello-Amoros M, Velez EJ, et al. (2020) Regulatory mechanisms involved in muscle and bone remodeling during refeeding in gilthead sea bream. Sci Rep 10, 184.

48. Larsson T, Morkore T, Kolstad K, et al. (2012) Gene expression profiling of soft and firm Atlantic salmon fillet. PLoS One 7, e39219.

49. Ou Y, Wang SJ, Li D, et al. (2016) Activation of SAT1 engages polyamine metabolism with p53-mediated ferroptotic responses. Proc Natl Acad Sci USA 113, E6806-E6812.

50. Dixon SJ \& Stockwell BR (2019) The Hallmarks of ferroptosis. Annu Rev Canc Biol 3, 35-54.

51. Stockwell BR, Angeli JPF, Bayir H, et al. (2017) Ferroptosis: a regulated cell death nexus linking metabolism, redox biology, and disease. Cell 171, 273-285.

52. Li J, Cao F, Yin HL, et al. (2020) Ferroptosis: past, present and future. Cell Death Dis 11, 1-13.

53. Arosio P, Ingrassia R \& Cavadini P (2009) Ferritins: a family of molecules for iron storage, antioxidation and more. Bba-Gen Subj 1790, 589-599.

54. Hou W, Xie Y, Song X, et al. (2016) Autophagy promotes ferroptosis by degradation of ferritin. Autophagy 12, $1425-1428$.

55. Yu XL \& Long YC (2016) Crosstalk between cystine and glutathione is critical for the regulation of amino acid signaling pathways and ferroptosis. Sci Rep-Uk 6, 1-11.
56. Murray Stewart T, Dunston TT, Woster PM, et al. (2018) Polyamine catabolism and oxidative damage. $\mathrm{J} \mathrm{Biol} \mathrm{Chem}$ 293, 18736-18745.

57. Tew KD, Manevich Y, Grek C, et al. (2011) The role of glutathione S-transferase $\mathrm{P}$ in signaling pathways and S-glutathionylation in cancer. Free Radical Bio Med 51, 299-313.

58. Anderson OS, Sant KE \& Dolinoy DC (2012) Nutrition and epigenetics: an interplay of dietary methyl donors, one-carbon metabolism and DNA methylation. J Nutr Biochem 23, 853-859.

59. Zhang N (2018) Role of methionine on epigenetic modification of DNA methylation and gene expression in animals. Anim Nutr 4, 11-16.

60. Serefidou M, Venkatasubramani AV \& Imhof A (2019) the impact of one carbon metabolism on histone methylation. Front Genet 10, 764

61. Wagner GR \& Hirschey MD (2014) Nonenzymatic protein acylation as a carbon stress regulated by sirtuin deacylases. Mol Cell 54, 5-16.

62. Nakagawa T, Lomb DJ, Haigis MC, et al. (2009) SIRT5 deacetylates carbamoyl phosphate synthetase 1 and regulates the urea cycle. Cell 137, 560-570.

63. Ali I, Conrad RJ, Verdin E, et al. (2018) Lysine acetylation goes global: from epigenetics to metabolism and therapeutics. Chem Rev 118, 340-376.

64. Sharples AP, Stewart CE \& Seaborne RA (2016) Does skeletal muscle have an 'epi'-memory? The role of epigenetics in nutritional programming, metabolic disease, aging and exercise. Aging Cell 15, 603-616.

65. Marousez L, Lesage J \& Eberle D (2019) Epigenetics: linking early postnatal nutrition to obesity programming? Nutrients 11, 2966.

66. Choudhary C, Kumar C, Gnad F, et al. (2009) Lysine acetylation targets protein complexes and co-regulates major cellular functions. Science 325, 834-840.

67. Saccone V \& Puri PL (2010) Epigenetic regulation of skeletal myogenesis. Organogenesis $\mathbf{6}, 48-53$.

68. Moghadam H, Morkore T \& Robinson N (2015) EpigeneticsPotential for programming fish for aquaculture? J Mar Sci Eng 3, 175-192.

69. Velez EJ, Lutfi E, Azizi S, et al. (2017) Understanding fish muscle growth regulation to optimize aquaculture production. Aquaculture 467, 28-40.

70. Panserat S, Marandel L, Seiliez I, et al. (2019) New insights on intermediary metabolism for a better understanding of nutrition in teleosts. Annu Rev Anim Biosci 7, 195-220.

71. Robinson DCL \& Dilworth FJ (2018) Epigenetic regulation of adult myogenesis. Curr Top Dev Biol 126, 235-284. 